\title{
Gallium Ga 68-NOTA-NFB
}

National Cancer Institute

\section{Source}

National Cancer Institute. Gallium Ga 68-NOTA-NFB. NCI Thesaurus. Code 120105.

A radioconjug ate composed of a derivative of the CXCR4 peptide antagonist T140, in which the N-terminal 4-fluoro-benzoyl group (NFB) is substituted with the chelating agent 1,4,7-triazacyclononane-N,N',N"-triacetic acid (NOTA) and radiolabeled with gallium Ga 68, with potential CXCR4 imaging activity during positron emission topography (PET). Upon administration, gallium Ga 68-NOTA-NFB selectively binds to CXCR4 and CXCR4-expressing tumor cells can then be visualized upon PET imaging. CXCR4, a chemokine receptor belonging to the G protein-coupled receptor (GPCR) gene family, plays an important role in chemotaxis and angiogenesis, and is upregulated in several tumor cell types. CXCR4 expression is correlated with tumor aggressiveness and high metastatic potential. Unmodified T140 binds non-specifically to red blood cells (RBCs); replacing the fluoro-benzoyl group with NOTA (NOTA-NFB) prevents the RBC binding almost entirely, while only minimally reducing the binding to CXCR4-positive tumor cells. 